\title{
Importance sampling for quantum Monte Carlo in manifolds: Addressing the time scale problem in simulations of molecular aggregates
}

\author{
T. Luan, ${ }^{1}$ E. Curotto, ${ }^{1, a)}$ and Massimo Mella ${ }^{2, b)}$ \\ ${ }^{1}$ Department of Chemistry and Physics, Arcadia University, Glenside, Pennsylvania 19038, USA \\ ${ }^{2}$ School of Chemistry, Cardiff University, Cardiff CF10 3AT, United Kingdom
}

(Received 24 December 2007; accepted 25 February 2008; published online 22 April 2008)

\begin{abstract}
Several importance sampling strategies are developed and tested for stereographic projection diffusion Monte Carlo in manifolds. We test a family of one parameter trial wavefunctions for variational Monte Carlo in stereographically projected manifolds which can be used to produce importance sampling. We use the double well potential in one dimensional Euclidean space to study systematically sampling issues for diffusion Monte Carlo. We find that diffusion Monte Carlo with importance sampling in manifolds is orders of magnitude more efficient compared to unguided diffusion Monte Carlo. Additionally, diffusion Monte Carlo with importance sampling in manifolds can overcome problems with nonconfining potentials and can suppress quasiergodicity effectively. We obtain the ground state energy and the wavefunction for the Stokmayer trimer. (C) 2008 American Institute of Physics. [DOI: 10.1063/1.2898539]
\end{abstract}

\section{INTRODUCTION}

In a recent article $^{1}$ one of our groups has developed several diffusion Monte Carlo (DMC) procedures to integrate the imaginary time Schrödinger equation in manifolds. Constraining intramolecular degrees of freedom has proven crucial in overcoming convergence difficulties with path integral simulations of molecular clusters. ${ }^{2-5}$ In DMC simulations of molecular aggregates, the degrees of freedom with high frequency require values of the imaginary time increment that are orders of magnitude smaller compared to those needed if such degrees of freedom are constrained. One expects massive efficiency improvements, assuming the adiabatic approximation is reasonably accurate. Therefore, DMC algorithms described in Ref. 1 can become potentially quite important in the advancement of our knowledge of finite molecular systems. However, at present, the stereographic projection diffusion Monte Carlo (SPDMC) methods described in Ref. 1 have been implemented only without importance sampling. ${ }^{6}$ In the present article we explore the possibility of extending some of the improvements proposed over the past three decades by the quantum Monte Carlo community. ${ }^{7-16}$ The majority of these developments have been for $n$-dimensional Euclidean spaces mapped by Cartesian coordinates, denoted $\mathbb{R}^{n}$ throughout. Their extension to generic manifolds is nontrivial, but is necessary for the successful implementation of SPDMC to realistic problems. Unlike alternative techniques recently reported in the literature, ${ }^{17-23}$ SPDMC methods avoid the need to compute Lagrange multipliers. Instead, the SPDMC methods are derived from the Schrödinger equation expressed in general manifolds using the machinery of differential geometry and tensor calculus; ${ }^{24-28}$ as such, they are interesting from the theoretical as much as the practical point of view.

\footnotetext{
a)Electronic mail: curotto@arcadia.edu.

${ }^{b)}$ Electronic mail: mellam@cardiff.ac.uk.
}

We envision employing SPDMC to determine the ground state properties of midsized molecular cluster, as, e.g., hydrogen bonded finite systems we have modeled recently in our groups, ${ }^{2}$ as a method complementary to the Monte Carlo path integral (MCPI). MCPI allows for thermal excitations to be considered, however, it is notoriously inefficient at temperatures where the ground state dominates statistically. In midsized molecular clusters we anticipate finding sampling problems, such as quasiergodicity. In DMC simulations the conventional wisdom is that particles tunnel through barriers, since DMC does replicate the "true quantum dynamics" of a system (in imaginary times). Years of research have helped us to establish this fact empirically for a number of diverse systems. ${ }^{29-35}$ In MCPI, quasiergodicity arises at the lowest temperatures, and one would naturally expect that the same takes place in DMC simulations at small values of the imaginary time increment. Therefore, the time scale problem for DMC simulations of molecular condensed matter in $\mathbb{R}^{n}$ could aggravate, if not create, quasiergodicity. This would make the case for holonomic constrains and SPDMC even more compelling. A number of simulations ${ }^{29-35}$ seem to suggest that in DMC, quasiergodicity can be suppressed by starting with a good approximation of the ground state distribution and using importance sampling (IS). However, quasiergodicity in DMC simulations, with or without IS, has never been explored systematically.

We organize the rest of the article in the following way. In Sec. II A, we derive the equivalent of the Schmoluchowski (Fokker-Planck) equation in manifolds. The equation is derived without assumptions on the form of the metric tensor, and it is applicable to all the cases of interest, including, e.g., the inertia ellipsoid for nonspherical rigid rotors, for which precession effects are captured by the off diagonal elements of the metric tensor. In Sec. II B we discuss the alternative IS strategy developed by Kalos et al. ${ }^{14}$ We refer to this technique as Green's function importance sampling 
(GFIS). In GFIS, rather than using a drift term to guide the diffusion, one modifies the relative branching weights after a regular diffusion step. In Sec. II B we show that the branching function for GFIS applies directly to general manifolds. There are no Jacobians in the branching function for GFIS, and this general result follows trivially by extending the definition of the Dirac delta function in generic manifolds. In Sec. II C, we comment on a possible trial wavefunction that can assist in both guiding SPDMC, and suppressing quasiergodicity at the same time. In Sec. III A we perform numerous tests in $\mathbb{R}^{1}$ with a double well potential. Solutions by DMC and by the discrete variable representation (DVR) for a large number of mass values and for two separate initial distributions of replicas are compared. The rationale behind these simulations is to determine systematically if quasiergodicity exists in DMC simulations, and if so, what are the conditions that worsen or ameliorate it. We also use the particle subjected to a double well potential in $\mathbb{R}^{1}$ to test the accuracy of the variational wavefunction proposed in Sec. II C. In Sec. III B we test the IS techniques for SPDMC outlined in Secs. II A and II B with the particle in a ring subject to a sinusoidal potential. Finally, in Sec. III C we demonstrate the applicability of IS-SPDMC to realistic molecular systems by determining the ground state properties of the Lennard-Jones dipole-dipole (LJDD) trimer, with a moderately elevated uniform dipole moment. Our conclusions are in Sec. IV.

\section{THEORY}

\section{A. The Schmoluchowski equation in manifolds}

The time dependent Schrödinger equation becomes a diffusion equation when expressed in imaginary time $\tau$ $=i t / \hbar$,

$$
\frac{\partial \psi}{\partial \tau}=\frac{\hbar^{2}}{2 \mu} \nabla^{2} \psi-V \psi
$$

Anderson used the isomorphism between the Schrödinger equation in imaginary time and the diffusion equation to develop a simulation process that yields the ground state energy and the wavefunction. ${ }^{6}$ The evolution of the system replicas, also known as psips, distributed according to some starting configuration can be carried out using Gaussian random numbers in $R^{n}$. The potential energy relative to some energy $E_{T}$ acts as the source or sink of particles.

$$
\frac{\partial \psi}{\partial \tau}=\frac{\hbar^{2}}{2 \mu} \nabla^{2} \psi-\left(V-E_{T}\right) \psi .
$$

The branching function

$$
w=\exp \left\{-\left(V-E_{T}\right) \Delta \tau\right\}
$$

produces a weight $w$ for the replica, and this is used to grow the replica (or annihilate it) in its present position.

Successive diffusion-branching iterations and adjustments to $E_{T}$ to maintain a constant population produce a sequence of distributions that approaches the ground state wavefunction asymptotically. The ground state energy is computed from the population average of $V$. Many improvements on the accuracy and efficiency of this process have been proposed. Some recent ones, using Itô calculus to produce second order schemes" and using "on the fly" extrapolations $^{8}$ to achieve third order convergence, have been developed in one of our groups. For bosonic ground states, for example, one can simply fix the branching process of the original scheme ${ }^{6}$ to produce second order convergence in $\Delta \tau .^{7}$ The new branching function is

$$
w=\exp \left\{-\frac{1}{2}\left(V+V_{\text {old }}-2 E_{T}\right) \Delta \tau\right\},
$$

where $V_{\text {old }}$ is the value of the potential for the replica before the diffusion and $V$ is the value of the potential for the system replica after the diffusion move is performed. Second order branching can produce converged energies with much larger values of $\Delta \tau$ improving the efficiency substantially. Another improvement is the use of guiding functions to produce importance sampling for the diffusion. Two formulations exist: The use of a guiding function to introduce drift into the diffusion process ${ }^{11-13}$ and the use of weighted branching derived from the Green function representation of the time evolution operator. ${ }^{14,15}$ Given a good approximation to the ground state $\psi_{T}(R)$ (usually obtained variationally), one lets $f(R, \tau)=\psi_{T}(R) \psi(R, \tau)$. Inserting this into the Schrödinger equation produces a diffusion equation for $f(R, \tau)$,

$$
\frac{\partial f}{\partial \tau}=\frac{\hbar^{2}}{2 \mu} \nabla^{2} f-\frac{\hbar^{2}}{\mu} \nabla\left(\frac{f \nabla\left[\psi_{T}\right]}{\psi_{T}}\right)-\left(E_{L}-E_{T}\right) f,
$$

where

$$
E_{L}=-\frac{\hbar^{2}}{\psi_{T} 2 \mu} \nabla^{2} \psi_{T}+V
$$

is the local energy, and

$$
\frac{\hbar^{2}}{\mu} \nabla\left(\frac{f \nabla\left[\psi_{T}\right]}{\psi_{T}}\right)
$$

is a drift term. Therefore, the diffusion process is for a density functional $f$ and it incorporates a velocity term with $\nabla\left[\psi_{T}\right] / \psi_{T}$ as a quantum force. Particular choices of the trial wavefunction can illustrate why the velocity term is referred to as a force with clarity. This fact is evidenced in Sec. II C where our choice of $\psi_{T}$ is introduced. Good trial wavefunctions can reduce the statistical error of the ground state energy for DMC by orders of magnitude. Additionally, optimized trial wavefunctions reduce the $\Delta \tau$ bias on the estimate of the ground state energy. This is not true for properties that depend only on the distribution, such as, e.g., structural properties.

The task before us is to find a general expression to include importance sampling in manifolds. We begin by deriving a general Fokker-Planck (Schmoluchowski) equation in non-Euclidean manifolds. Melik-Alaverdian et al. $^{23}$ have recently succeeded in producing an importance sampling DMC equation with drift for the many electrons on the Haldene sphere, a curved space that arises in the study of the fractional quantum Hall effect. However, the authors of Ref. 23 use a special property of the metric tensor making their treatment insufficiently general for applications in molecular physics with holonomic constraints. 
The Schrödinger equation ${ }^{26-28}$ in a manifold endowed with a metric ${ }^{24,25} g_{\mu \nu}$ reads

$$
-i \hbar \frac{\partial \psi}{\partial t}=-\frac{\hbar^{2}}{2} \Delta_{\mathrm{LB}} \psi+\left(V-E_{T}\right) \psi .
$$

$\Delta_{\text {LB }}$ is the Laplace-Beltrami operator, ${ }^{26-28}$

$$
\Delta_{\mathrm{LB}}=\frac{1}{\sqrt{g}} \partial_{\nu} g^{\mu \nu} \sqrt{g} \partial_{\mu},
$$

where $g^{\mu \nu}$ is the inverse of the metric tensor and $g$ $=\operatorname{det}\left(g_{\mu \nu}\right)$. In imaginary time $\tau=i t / \hbar$, the Schrödinger equation becomes

$$
\frac{\partial \psi}{\partial \tau}=\frac{\hbar^{2}}{2} \Delta_{\mathrm{LB}} \psi-\left(V-E_{T}\right) \psi
$$

It is possible to simulate Eq. (10) in manifolds. Unlike its counterpart in flat space, the steps used to simulate diffusion in the manifold do not generally have a Gaussian distribution, since the metric tensor $g_{\mu \nu}$ in the detailed balance expression,

$$
\begin{aligned}
& T(\Delta q) \exp \left(-\frac{g_{\mu \nu}(q) \Delta q^{\mu} \Delta q^{\nu}}{2}\right) \\
& \rightarrow T\left(\Delta q^{\prime}\right) \exp \left(-\frac{g_{\mu \nu}\left(q^{\prime}\right)\left(\Delta q^{\mu}\right)^{\prime}\left(\Delta q^{\nu}\right)^{\prime}}{2}\right),
\end{aligned}
$$

normally depends on the configuration. In Ref. 1 we propose three algorithms that address the configurational dependence of the metric tensor during the generation on random steps $\Delta q^{\mu}$ by the rejection technique. These are based on three choices for the configuration $q$ that enters into the acceptance-rejection decisions. The DMC algorithms of Ref. 1 are generally applicable provided that (a) the metric tensor $g_{\mu \nu}$ is expressible as a function of the configuration. (b) The space is mappable with a single stereographic projection map. The second condition is restrictive, but necessary to avoid the use of boundary conditions in stochastic simulations. Boundary conditions are primarily employed to remove edge effects in Euclidean space simulations of bulk matter, they do not function as expected when more complicated topologies are mapped with open sets (e.g., the angles $\phi$ for the particle in a ring). We have shown in a number of $\operatorname{articles}^{2-4}$ that the spaces of interest to molecular physicists, produced by imposing holonomic constraints in molecular systems, can be mapped with stereographic projections; these include the inertia ellipsoids for linear and nonlinear rigid tops, torsional, bending, and radial degrees of freedom. ${ }^{1}$

It is also possible to find a variational solution for Eq. (10). We return to how one could construct such variational solution in manifolds later in the article. Let us assume that $\psi_{T}$ is such optimized solution, and let us insert $f(q, \tau)$ $=\psi_{T}(q) \psi(q, \tau)$ into Eq. (10),

$$
\frac{\partial f}{\partial \tau}=\frac{\hbar^{2}}{2} \frac{\psi_{T}}{\sqrt{g}} \partial_{\nu} g^{\mu \nu} \sqrt{g}\left\{\frac{1}{\psi_{T}} \partial_{\mu}[f]-\frac{f}{\psi_{T}^{2}} \partial_{\mu}\left[\psi_{T}\right]\right\}-\left(V-E_{T}\right) f .
$$

Using the product rule to break up the two terms of the Laplace-Beltrami operator, and simplifying we obtain

$$
\begin{aligned}
\frac{\partial f}{\partial \tau}= & \frac{\hbar^{2}}{2} \Delta_{\mathrm{LB}} f-\hbar^{2} g^{\mu \nu} \partial_{\nu}\left(\frac{f \partial_{\mu}\left[\psi_{T}\right]}{\psi_{T}}\right) \\
& -\frac{\hbar^{2} f}{2 \psi_{T} \sqrt{g}}\left(\partial_{\mu}\left[\psi_{T}\right]\right) \partial_{\nu}\left[g^{\mu \nu} \sqrt{g}\right] \\
& +\frac{\hbar^{2} f}{2 \psi_{T}} g^{\mu \nu} \partial_{\nu} \partial_{\mu}\left[\psi_{T}\right]-\left(V-E_{T}\right) f .
\end{aligned}
$$

The second term on the right hand side is the metric space equivalent of the drift

$$
F_{\nu}^{(Q)}=\left(\frac{f \partial_{\nu}\left[\psi_{T}\right]}{\psi_{T}}\right) .
$$

The expression in Eq. (14) is exactly the same expression for the quantum force, ${ }^{13}$ obtained in $\mathbb{R}^{n}$. The third and fourth terms in Eq. (13) are parts of the Laplace-Beltrami operator acting on $\psi_{T}$. To obtain the equivalent of the local energy term one must recombine these into a local energy term. However, inspection of Eq. (9) shows that the third term in Eq. (13) has the wrong sign. Adding and subtracting twice the additive inverse of the third term on the right hand side and collecting gives the final result,

$$
\frac{\partial f}{\partial \tau}=\frac{\hbar^{2}}{2}\left(\Delta_{\mathrm{LB}}+W_{T}\right) f-\hbar^{2} \nabla^{\nu} F_{\nu}^{(Q)}-\left(E_{L}-E_{T}\right) f,
$$

where $\nabla^{\nu}=g^{\mu \nu} \partial_{\mu}$ is the gradient with the raised index. The local energy $E_{L}$ is the general metric space equivalent of the usual local energy,

$$
E_{L}=-\frac{\hbar^{2}}{2 \psi_{T}} \Delta_{\mathrm{LB}} \psi_{T}+V
$$

and

$$
W_{T}=\frac{2}{\psi_{T} \sqrt{g}}\left(\partial_{\mu}\left[\psi_{T}\right]\right) \partial_{\nu}\left[g^{\mu \nu} \sqrt{g}\right]
$$

is interpreted as an added geometric modification of the diffusion operator. $W_{T}$ vanishes in the limit of a constant trial wavefunction. There are no other plausible interpretations for $W_{T}$; it cannot be interpreted as as quantum potential, for example, since the local energy estimator must be composed of the potential energy and the Laplace-Beltrami operator alone. $W_{T}$ is independent of the diffusion steps, but it can enter into the Metropolis algorithm when generating steps from the correct distribution, if a strategy implemented from Ref. 1 is such that $q^{\prime} \neq q$ in Eq. (11).

\section{B. Importance sampling in manifolds with Green's functions}

Green's function Monte Carlo method ${ }^{14,15}$ with importance sampling is as effective as the drift method and quite simple to implement. Let us consider the integral representation of the time dependent Schrödinger equation,

$$
\psi(q, \tau+\Delta \tau)=\int_{\mathbb{M}} G\left(q, q^{\prime}, \Delta \tau\right) \psi\left(q^{\prime}, \tau\right) d q^{\prime} .
$$

$G\left(q, q^{\prime}, \Delta \tau\right)$ satisfies the time dependent Schrödinger equation and satisfies the initial condition 


$$
\lim _{\Delta \tau \rightarrow 0} G\left(q, q^{\prime}, \Delta \tau\right)=\delta\left(q-q^{\prime}\right) .
$$

The reader should note that the Jacobian is absent in Eq. (18) as a result of the definition of the Dirac delta function in manifolds. ${ }^{36}$

$$
\int_{\mathbb{M}} \delta\left(q-q^{\prime}\right) \psi\left(q^{\prime}, \tau\right) d q^{\prime}=\psi(q, t) .
$$

Clearly, the argument of the integral in Eq. (20) cannot contain a Jacobian.

Equation (18) can be solved by approximating the Green function locally. To first order in $\Delta \tau$ we have

$$
G\left(q, q^{\prime}, \tau\right) \approx \exp \left(-\frac{g_{\mu \nu} \Delta q^{\mu} \Delta q^{\nu}}{2} \Delta \tau\right) \exp \left[-\left(V-V_{\text {ref }}\right) \Delta \tau\right] .
$$

To simulate the population evolution using Eq. (21), one could create an algorithm that performs random moves for each replica with $\Delta q$ distributed according to the first exponential on the left hand side, and then branch using the second exponential. The resulting algorithms would be identical to those we developed in Ref. 1 without importance sampling. However, with an approximate wavefunction we can modify both sides of Eq. (18),

$$
\psi_{T}(q) \psi(q, \tau+\Delta \tau)=\int_{\mathbb{M}} G\left(q, q^{\prime}, \Delta \tau\right) \psi_{T}\left(q^{\prime}\right) \psi\left(q^{\prime}, \tau\right) d q,
$$

which rearranges to

$$
\psi(q, \tau+\Delta \tau)=\int_{\mathbb{M}} G\left(q, q^{\prime}, \tau\right) \frac{\psi_{T}\left(q^{\prime}\right)}{\psi_{T}(q)} \psi\left(q^{\prime}, \tau\right) d q .
$$

Using Eq. (21) we approximate the kernel of the integral equations in Eq. (23) with

$$
\exp \left(-\frac{g_{\mu \nu} \Delta q^{\mu} \Delta q^{\nu}}{2} \Delta \tau\right) \frac{\psi_{T}(q)}{\psi_{T}(q+\Delta q)} \exp \left[-\left(V-V_{\text {ref }}\right) \Delta \tau\right] .
$$

Therefore, we move each replica with $\Delta q$ distributed according to the first exponential, and then we subject each replica to a branching process guided by the following function:

$$
w_{i}=\frac{\psi_{T}(q)}{\psi_{T}(q+\Delta q)} \exp \left[-\left(V-V_{\mathrm{ref}}\right) \Delta \tau\right] .
$$

An equivalent weighted second order branching function can be derived as well. ${ }^{7}$ The only difference for manifolds is that the ground state energy (used to fix the reference energy $E_{T}$ ) is obtained by averaging $E_{L}$, given by Eq. (16).

At this stage, few comments may be useful to clarify the impact of using Green's function Monte Carlo approach of Eq. (25) instead of the more commonly employed Schmoluchowski formulation provided in Eqs. (5) and (15). Implementations of Eq. (25), just like unguided DMC, may suffer from population explosion when potentials unbounded from below are used in the simulation. This is corrected in Eqs. (5) and (15) by using $E_{L}$ in conjunction with a $\psi_{T}$ that satisfies the analytical properties of the exact ground state in the regions where the potential diverges (e.g., at the coalescence point in Coulomb systems). This is, however, not necessary when simulating a molecular aggregate, whose interaction potential usually is bounded from below. Besides, numerical experiments and theoretical analysis ${ }^{10}$ have shown that the usage of Eq. (5) as an importance sampling device may worsen the time step bias due to a large discretization error in the integration of its diffusion-drift part when simulating atomic clusters. Whether or not this is also the case in curved manifolds, is an issue that we aim to investigate in this work.

\section{Variational Monte Carlo (VMC) in manifolds}

The strategies developed in Secs. II A and II B need a trial wavefunction in MI. In this article we explore one possibility, which, at least in a formal sense, holds the promise to assist not only in improving the efficiency of SPDMC, but also to overcome most sampling problems that arise from quasiergodicity. The trial function we propose is

$$
\psi_{\beta}(q)=A \exp \{-1 / 2 \beta V(q)\} .
$$

This choice is calculated to be among the best for manifolds for the following reason. The variational energy,

$$
E_{\beta}=\frac{\int_{\mathbb{N}} d q g^{1 / 2}\left(\psi_{\beta}^{-1} \hat{H} \psi_{\beta}\right)\left|\psi_{\beta}\right|^{2}}{\int_{\mathbb{M}} d q g^{1 / 2}\left|\psi_{\beta}\right|^{2}},
$$

can be computed with parallel tempering ${ }^{37-40}$ for various values of $\beta$ accomplishing the optimization of the parameter $\beta$ and ensuring ergodic sampling in $\mathrm{MI}$ at the same time. The type of trial wavefunction we propose in Eq. (26) provides a clear interpretation of the drift term that is derived in flat, and, in the present work, in curved manifolds. From Eq. (26) is it follows that

$$
\psi_{\beta}(q)^{-1} \partial_{\nu} \psi_{\beta}(q)=-\frac{1}{2} \beta \partial_{\nu} V .
$$

\section{RESULTS}

\section{A. Double well in $\mathbb{R}^{1}$}

The double well potential model is a quartic polynomial that has been proposed by Frantz ${ }^{41}$ to study quasiergodicity in stochastic simulations.

$$
V(x)=\frac{3}{2 \alpha+1} x^{4}+\frac{4 \alpha-4}{2 \alpha+1} x^{3}-\frac{6 \alpha}{2 \alpha+1} x^{2}+1 .
$$

The potential has a global minimum at $x=1$ where $V=0$, a local minimum $V=1-\gamma$ at $x=-\alpha$, and a barrier of 1 hartree in between. The $V=1$ local maximum is at $x=0$. Given a value of $\gamma$, one computes $\alpha$ recursively from

$$
\gamma=\alpha^{3}\left(\frac{\alpha+2}{2 \alpha+1}\right) \text {. }
$$

For this investigation we use $\gamma=0.9$, the value of $\alpha$ is approximately 0.9613 . To determine if DMC suffers from quasiergodicity, we use simulations for a range of mass values starting from two separate delta distributions of psips: $\psi(x, \tau=0)=\delta(x-1)$ and $\psi(x, \tau=0)=\delta(x+\alpha)$. These correspond to all psips beginning at the global minimum $x=1$ and 

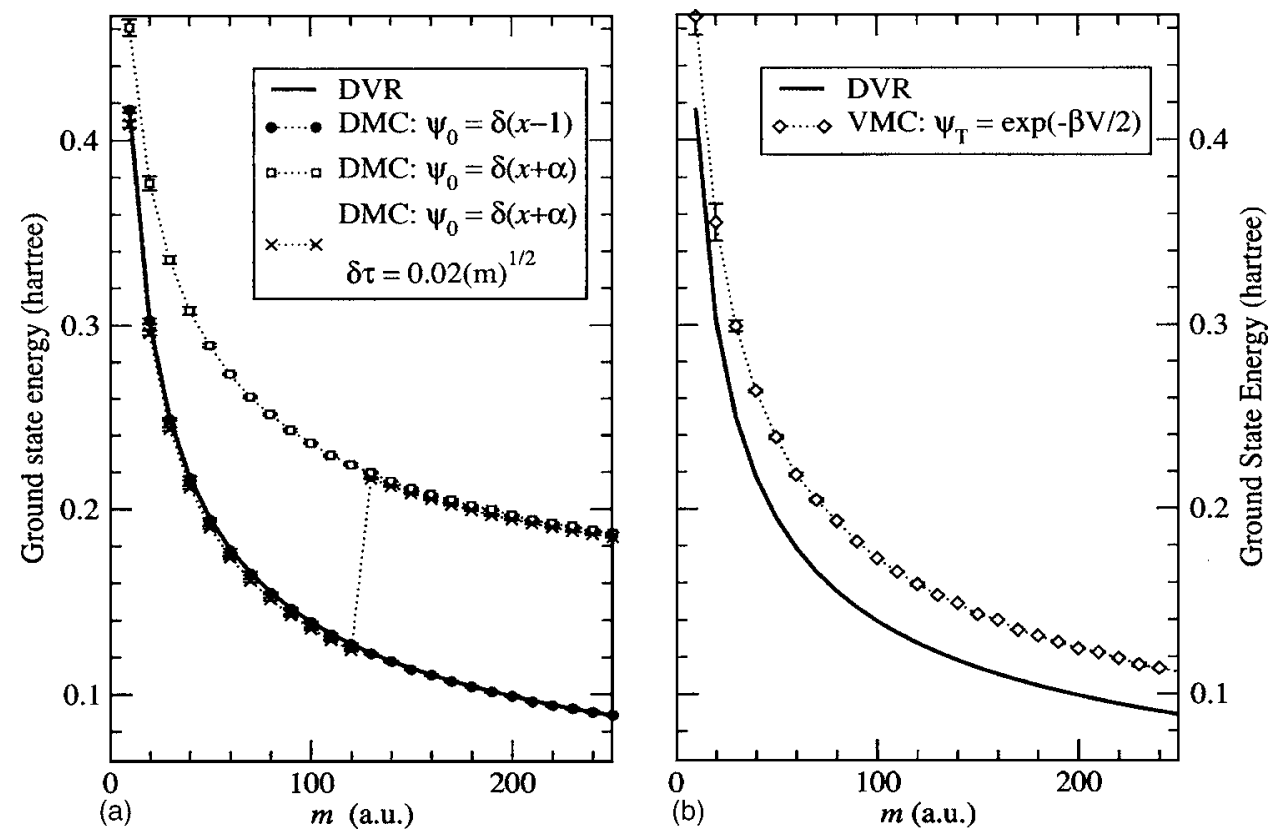

FIG. 1. (a) Ground state energy of a particle of mass $m$ in a double well [cf. Eq. (29)]. The solid line represents the exact value obtained by diagonalization. The black circles are DMC simulations obtained by starting the diffusion from a delta distribution centered at the global minimum. The points marked by white squares and by $\times$ are DMC simulations initiated from a $\delta$ distribution centered at the local minimum with two sets of values of $\Delta \tau$. (b) Comparison between the variational [cf. Eq. (27)] and the exact ground state energy for the particle in $\mathbb{R}^{1}$ trapped in a double well potential.

at the local minimum $x=-\alpha$, respectively. The masses range from 10 to 500 a.u. We step through the values of mass systematically in increments of 10 a.u.

Each DMC simulation consists of 5000 moves with populations of $N \approx 10^{5}$ psips. The value of $\Delta \tau$ is adjusted for every mass value according to

$$
\Delta \tau=0.002(m)^{1 / 2} \text {. }
$$

The value of 0.002 is determined empirically by checking the convergence behavior of DMC at both extremes of the mass range starting DMC from the global minimum distribution, $\psi(x, \tau=0)=\delta(x-1)$ in a number of test runs. During these test runs, we find that with the chosen values of $\Delta \tau$ the energy approaches a plateau around the exact value after about 500 moves. Nevertheless, we only average energy data in the last 1000 moves. The ground state energy is compared with a vector space computation. ${ }^{42}$ The DVR is constructed in $-2.3 \leqslant x \leqslant+2.3$ with 1000 points.

The results are displayed in Fig. 1(a). At a mass of about 200 units, the exact ground state energy drops below 0.1 hartree, making the second well classically forbidden. The dark circles are obtained from unguided DMC simulations with the initial condition $\psi(x, \tau=0)=\delta(x-1)$; the agreement between DMC and the exact ground state energy in this case is excellent. Close analysis shows that the DMC data with the global minimum as a starting configuration and DVR results agree within the statistical error at all the values of the mass. Drastically different results are obtained by switching the initial condition for the diffusion to $\psi(x, \tau$ $=0)=\delta(x+\alpha)$. Data marked with $\times$ 's in Fig. 1(a) represent average energies obtained by initiating DMC with $\psi(x, \tau$ $=0)=\delta(x+\alpha)$ but using a time step ten times larger than the white squares, namely, $\Delta \tau$ is adjusted for every mass value according to $\Delta \tau=0.02(\mathrm{~m})^{1 / 2}$.

Distributions of psips beginning in the local well for masses greater than 200 a.u. should, according to conventional wisdom, tunnel out from the higher energy well entirely. Clearly, Fig. 1(a) indicates otherwise. To better inter- pret the results in Fig. 1(a), it is necessary to investigate more closely the exact ground state wavefunctions and then the DMC simulations with the local minimum as the starting configuration. In Fig. 2 we present a composite sketch of the wavefunctions obtained from DMC and DVR (solid line) for a mass of 10 a.u. For masses in between 10 and 200 a.u., the DVR wavefunction is heavily skewed toward the global minimum despite the relatively large value of the ground state energy compared to the energy difference between the two wells at small $m$. The DMC wavefunctions are obtained by normalizing the psip histogram for the population after 5000 moves. Both DMC wavefunctions are obtained by starting the diffusion from the high energy well: $\psi(x, \tau=0)$ $=\delta(x+\alpha)$. The white squares represent the wavefunction after 300 a.u. of time, whereas the white circles are obtained after 30 a.u. of time. Thus, tunneling does occur as conventional wisdom has it, but even for the lightest mass investigated, the adjustment of the wavefunction takes place quite slowly. Increasing the mass worsens the problem and at a sufficiently large mass, even the larger time steps fail to produce a good wavefunction as Fig. 1 clearly demonstrates.

It is an insightful exercise to measure the "tunneling time" in the double well as a function of the particle mass when its starting wavefunction is a Dirac delta at $-\alpha$. Additionally, we find the comparison of the tunneling times for the unguided DMC (Ref. 6) method and IS-DMC instructive. In both cases the initial wavefunction remains $\psi(x, \tau=0)$ $=\delta(x+\alpha)$, rather than choosing an initial population distributed as $\psi_{T}$, as normally practiced. Parallel tempering simulations are employed to span a large set of parameter values [ $\beta$ in Eq. (27)] at every value of the mass. $1 \times 10^{6}$ moves to equilibrate is followed by another million moves to average the variational estimate of the ground state, at approximately 40 different values of the parameter $\beta$. The optimal VMC energy for several values of the mass is compared against the exact energy in Fig. 1(b). The error bars are estimated from the energy fluctuations between neighboring values of $\beta$. We define the tunneling time as the total time $M \Delta \tau$ required by 


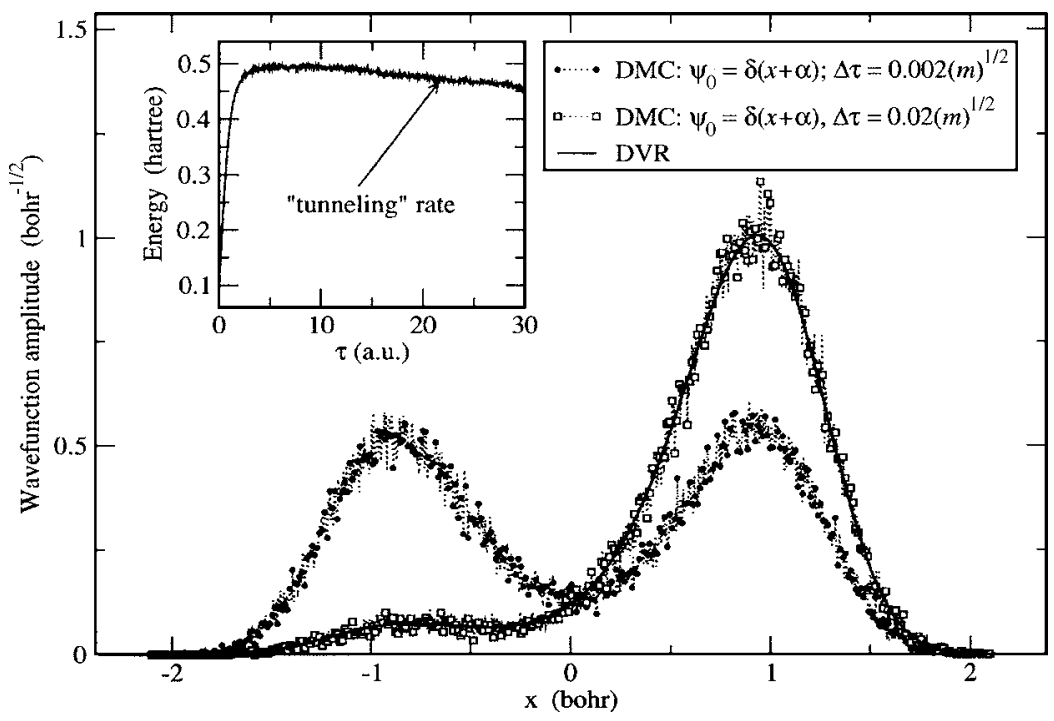

FIG. 2. Ground state wavefunctions for a particle with 10 a.u. of mass in the double well [cf. Eq. (29)] with the DVR (solid black line), with DMC after 5000 moves starting from the left well. $\Delta \tau=0.00632$ hartree $^{-1}$ are the black circles, and $\Delta \tau=0.0632$ hartree $^{-1}$ are the white squares. Insert: Population average energy as a function of imaginary time for a particle with 10 a.u. of mass in the double well. The simulated diffusion is initiated from the high energy well with $\psi(x, \tau=0)=\delta(x$ $+\alpha)$ as the initial distribution.

the algorithm to produce a population average energy within $5 \%$ of its exact value. $M$ is the number of iterations, and $\Delta \tau$ is computed with Eq. (31). The results for the unguided DMC (Ref. 6) are the white circles in Fig. 3. The unguided DMC is essentially trapped in the high energy well at masses above 130 a.u., where the tunneling times are practically incommensurate. Adding IS [c.f. Eq. (25) and the black squares in Fig. 3] seems to diminish the problem substantially. The tunneling times for IS-DMC can be measured even for the extreme case of a system starting in a classically forbidden well (not shown in Fig. 3). For a mass of 230 a.u., for example, the time to tunnel out from the upper well grows to $\approx 7 \times 10^{4}$ hartree $^{-1}$.

The definition of IS tunneling times, as the total time $M \Delta \tau$ required by the algorithm to produce a population average energy within $5 \%$ of its exact value in this particular case, is valid, since the optimized variational wavefunction is not very accurate. Had the variational energy been accurate to better than $5 \%$, then, is would have been impossible to compare guided and unguided tunneling times. As Fig. 1(b) shows, the variational energies deviate from the exact values by an amount in between $9 \%$ and $11 \%$. The optimized variational wavefunction overestimates the importance of the sec- ond well, as it displays a more intense peak at the local minimum when compared with the converged DVR wavefunctions. To gain a deeper understanding of how the trial wavefunction assists in the tunneling process for the diffusion initiated in the local minimum with $\psi(x, \tau=0)=\delta(x$ $+\alpha$ ), we repeat the calculations monitoring the energy ratio $r_{e}$

$$
r_{e}=\frac{\left|E-E_{\text {exact }}\right|}{\left|E_{\beta}-E_{\text {exact }}\right|},
$$

where $E, E_{\text {exact }}$, and $E_{\beta}$ are, respectively, the DMC population average energy, the exact ground state obtained by diagonalization, and the variational energy obtained from the optimized trial wavefunction. Defining the tunneling time as the time it takes for $r_{e}$ to drop below $40 \%$ produces results comparable to those graphed in Fig. 3. The value of $40 \%$ is obtained by combining the arbitrary threshold of $5 \%$ chosen for the unguided definition and the fact that $E_{\beta}$ is about $11 \%$ accurate. $r_{e}$ as a function of time for several values of mass is graphed in Fig. 4. The data in Fig. 4 yield additional insight into the tunneling process. $r_{e}$ spikes well above one during the first few diffusion steps and drops below 1 rather quickly

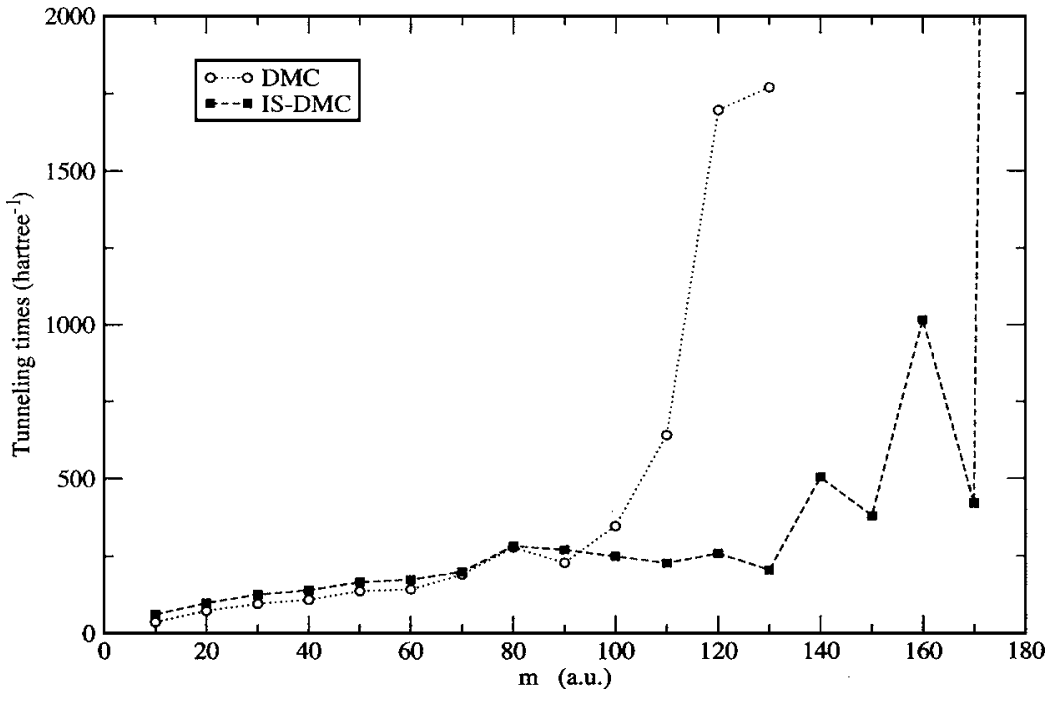

FIG. 3. The total time (in hartree ${ }^{-1}$ ) required for a replica population to diffuse through the barrier between the high energy well and the lower one to achieve an average ground state energy within $5 \%$ of the exact value. The systems are particles in a double well in $\mathbb{R}^{1}$, with initial wavefunction a Dirac delta at $x=-\alpha$. 


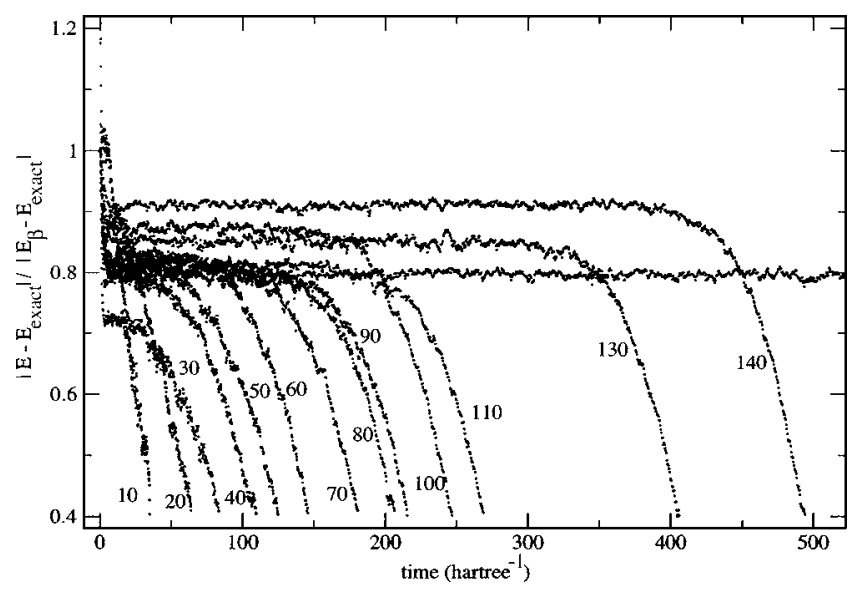

FIG. 4. The energy ratio $r_{e}$ [cf. Eq. (32)] as a function of total diffusion time for several values of mass as the replica population diffuses through the barrier between the high energy well and the lower one, in a double well in $\mathrm{R}^{1}$, with the initial wavefunction a Dirac delta at $x=-\alpha$.

as the result of adjustments in $E_{T}$. At masses of 40 a.u. and above, the value of $r_{e}$ remains flat around 0.8 for a period of time proportional to the mass of the particle. This time is inversely proportional to the probability that a diffusion step takes one psip from the high energy well into the lower energy one. As soon as one psip has moved into the lower energy well, branching takes over, the wavefunction grows in the correct part of configuration space, and the rate at which successive populations adjust the energy becomes independent of mass. Clearly, initiating the DMC walk using the trial wavefunction to obtain a starting distribution, as commonly practiced, eliminates any mass dependence on the tunneling time scale.

\section{B. The particle in $\mathrm{S}^{1}$}

The particle in a ring is used to test Eqs. (15), (16), and (25), in conjunction with the rejection methods used to produce distributions of $\Delta q^{\mu}$ for the diffusion part of the propagation according to

$$
p(\Delta q, \Delta \tau) \propto \exp \left(-\frac{q_{\mu \nu} \Delta q^{\mu} \Delta q^{\nu}}{2 \Delta \tau}\right) .
$$

The topological information of the space is contained in the metric tensor $g_{\mu \nu}$, which, in general, depends on the configuration. $g_{\mu \nu}$ is represented by a $n \times n$ symmetric matrix of functions. For $\mathbb{R}^{n}, g_{\mu \nu}=m \delta_{\mu \nu}$, with $m$ the mass, and Eq. (33) becomes the familiar Gaussian distribution with $\sigma^{2}=\Delta \tau / m$. The configuration dependence of $p(\Delta q, \Delta \tau)$ produces an additional challenge, since information about the location of the replica has to enter into the algorithm, as it produces random variables $\Delta q$ distributed according to Eq. (33), before the replica can be moved. This issue was addressed in detail in a recent article. ${ }^{1}$ We reproduce the numerical procedure that we use in the present study for convenience.

(I) Step 1: Draw $n$ random numbers in $[0,1]$ store them in the vector $\eta$ and compute $\Delta q^{\prime}$ with
$\Delta q^{\prime}=\Delta q+\gamma(\eta-0.5)$

$\gamma$ is adjusted to produce a $50 \%$ rejection rate, as commonly practiced with rejection techniques.

(II) Step 2: Using $\Delta q$ from the previous replica and letting $q^{\prime}=q$ be the configuration of the present replica compute the acceptance probability $P\left(\Delta q \rightarrow \Delta q^{\prime}\right)$ for $\Delta q^{\prime}$

$$
\begin{aligned}
P(\Delta q \rightarrow & \left.\Delta q^{\prime}\right)=\min \left\{1, \exp \left[-\frac{g_{\mu \nu}\left(q^{\prime}\right)\left(\Delta q^{\mu}\right)^{\prime}\left(\Delta q^{\nu}\right)^{\prime}}{2 \Delta \tau}\right.\right. \\
& \left.\left.+\frac{g_{\mu \nu}(q) \Delta q^{\mu} \Delta q^{\nu}}{2 \Delta \tau}\right]\right\} .
\end{aligned}
$$

(III) Step 3: Move the replica with $q+\Delta q^{\prime} \rightarrow q^{\prime}$ if the move is accepted or with $q+\Delta q \rightarrow q^{\prime}$ if rejected.

(IV) Step 4: Set $\Delta q^{\prime} \rightarrow \Delta q$ if the move is accepted and repeat from step 1 for all the replicas in the population.

This is one of the three methods that are tested and are confirmed to produce the correct asymptotic distributions for the ground state wavefunction and for the steps $\Delta q$ in Ref. 1 . Note that with the choice $q^{\prime}=q$ in step 2, the term we name $W_{T}$ in Eq. (17) vanishes from the probability $P\left(\Delta q \rightarrow \Delta q^{\prime}\right)$. The space $\mathrm{S}^{1}$ is a set of points $p(x, y)$, such that

$$
x^{2}+y^{2}=R^{2},
$$

where $R$ is the radius. $S^{1}$ is a subset of the points in a plane $(x, y) \in \mathbb{R}^{2}$. The stereographic projection map $\Phi_{\xi}: \mathbb{R}^{2} \rightarrow S^{1}$ and its inverse,

$$
x=\frac{4 R^{2} \xi}{\xi^{2}+4 R^{2}}, \quad y=R \frac{\xi^{2}-4 R^{2}}{\xi^{2}+4 R^{2}},
$$

are trivial to derive. ${ }^{5}$ Using Eq. (37), and the transformation law for 2 -forms, ${ }^{24}$ on $g_{\mu^{\prime} \nu^{\prime}}=m \delta_{\mu^{\prime} \nu^{\prime}}$ in $\mathbb{R}^{2}$, one obtains the metric tensor element

$$
g=\frac{16 m R^{2}}{\left(\xi^{2}+4 R^{2}\right)^{2}} .
$$

The mass $m$ is 500 a.u., the radius $R$ is set to $1 \mathrm{bohr}$, and the potential energy surface is nonconfining,

$$
V=\frac{4 \xi}{\xi^{2}+4} \text {. }
$$

The potential function has a minimum value of -1 at $\xi=-2$, and a maximum of +1 at $\xi=+2 ; V$ tends to zero at both asymptotes. The right hand side of Eq. (39) transforms to $\cos \theta$. The exact ground state can be obtained by diagonalizing the following Hamiltonian matrix:

$$
H_{i j}=\frac{\hbar^{2}}{2 m R^{2}} \delta_{i j}+\frac{1}{2}\left(\delta_{i j+1}+\delta_{i j-1}\right) .
$$

The ground state energy obtained by diagonalization is $\left(-0.977701995 \pm 2 \times 10^{-9}\right)$ hartree, where the uncertainty is obtained by estimating the basis set truncation error. The variational ground state is $\left(-0.9777014 \pm 2 \times 10^{-7}\right)$ hartree, in excellent agreement with the exact result, and is obtained by optimizing $\beta$ to 11.244 hartree $^{-1}$ [c.f. Eq. (26)]. To optimize $\beta$ we compute the integral in Eq. (27), using regular 

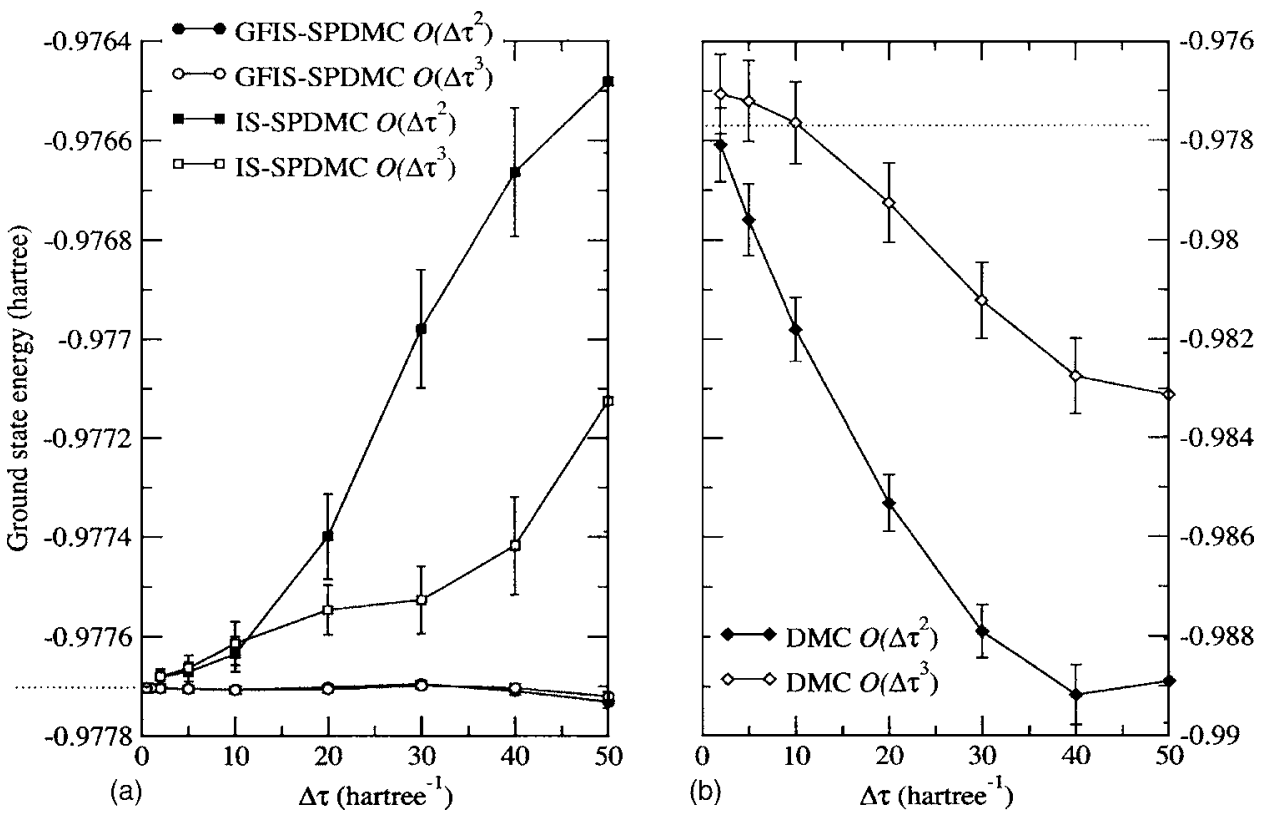

FIG. 5. Ground state energies for a particle of 500 a.u. of mass in a ring of 1 bohr, subjected to a sinusoidal potential (in terms of the angle). The ground state energies are obtained with six different styles of SPDMC simulations for a number of step sizes $\Delta \tau$. (a) GFIS-SPDMC with first order branching (black circles) is compared with GFIS-SPDMC second order branching (white circles) and with the drift IS methods [first order branching (black squares), second order branching (white squares)]. (b) Unguided SPDMC with first and second order branching. The exact ground state energy is shown with a dotted line in both graphs.

Metropolis with $10^{6}$ "warm-up" moves followed by $10^{6}$ moves to compute the average. The statistical error is estimated by comparing neighboring values of the variational energy. Likely, the level of agreement of the variational result is fortuitous, given the simplicity of the system. In Fig. 5 we present a summary of six styles of DMC simulations for the particle in a ring at a number of $\Delta \tau$ values. All the data points refer to estimates of the ground state energy, and these are assembled in the same graph to display most evidently the level of efficiency and convergence behavior of each style. The best convergence and most efficient simulations are clearly the GFIS-SPDMC simulations, from which we obtain a ground state energy of $\left(-0.977703 \pm 4 \times 10^{-6}\right)$. These results are graphed in Fig. 5(a) with black circles to represent GFIS simulations with first order branching and white circles to represent GFIS simulations with second order branching,

$$
w=\frac{\psi_{T}\left(q^{\prime}\right)}{\psi_{T}(q)} \exp \left\{-\frac{1}{2}\left[V(q)+V\left(q^{\prime}\right)-2 E_{T}\right] \Delta \tau\right\} .
$$

On the scale of the graph these sets of points are indistinguishable. The error bars obtained by performing block averages and computing a 95\% confidence interval are smaller than the symbols. The black squares and the white squares represent IS results obtained with Eq. (15), with, respectively, first order and second order expressions for the weights. The IS-SPDMC results with the Schmoluchowski propagator are clearly converging to the same ground state more efficiently than the unguided SPDMC [black and white diamonds for first order and second order branching, respectively, in Fig. 5(b)], but less efficiently than the GFIS simulations. It is important to note that both IS strategies are capable of controlling the effects of the nonconfining poten- tial, contrary to what occurs for the two unguided SPDMC simulations, which overestimate the ground state energy by a small amount. This can be seen with the points marked by the white diamonds in Fig. 5(b), where the asymptote for this set of points appears different. We increase the sample size to reduce the statistical error of the unguided SPDMC, and we find that the difference between the converged ground state and its exact value is small but significant. This type of problem has been reported in other simulations with nonconfining potentials. ${ }^{9}$ IS successfully eliminates dissociative effects even in curved spaces.

In addition, we perform a number of extrapolations using both the on the fly and a posteriori approaches. In both cases we learn that fitting the GFIS and the drift IS models with first order branching produces the exact result, whereas when we try extrapolating GFIS with second order branching using a second order extrapolation scheme, we find small but significant differences. These results indicate that while the branching part is rigorously second order, the diffusion part of the algorithms still produces a sequence of distributions that converge to the ground state wavefunction to first order. The presence of a configuration dependence in the distribution of the diffusion steps apparently spoils the exact solution of Eq. (18), the same way that it happens with the drift term in the Fokker-Planck operator. ${ }^{7}$

\section{Ground state properties of the LJDD trimer}

Stokmayer fluids ${ }^{43-49}$ are composed of point dipoles with an additional Lennard-Jones interaction among pairs of points. The majority of the theoretical work on Stokmayer fluids has focused on bulk properties. In recent works we have developed extensions of the genetic algorithm and par- 
allel tempering to study the thermodynamics of a cluster of Lennard-Jones point dipoles. ${ }^{48,49}$ For a finite $n$ point dipole cluster the potential energy is

$$
V=\sum_{i, j=1(j>i)}^{n}\left(V_{i j}^{(D D)}+V_{i j}^{(L J)}\right)+V_{c} .
$$

The Lennard-Jones potential contains two parameters,

$$
V_{i j}^{(L J)}=4 \epsilon\left[\left(\frac{r_{0}}{r_{i j}}\right)^{12}-\left(\frac{r_{0}}{r_{i j}}\right)^{6}\right],
$$

where $r_{0}$ is the length scale and $\epsilon$ is the energy scale. Let $R$ represent the size of the dipole $\mu_{D}=R q, \xi_{i}^{\mu} \mu=1,2$ the stereographic projections for center $i$, and $\sigma_{i}=\left(\xi_{i}^{1}\right)^{2}+\left(\xi_{i}^{2}\right)^{2}$. The dipole-dipole interaction expressed in terms of stereographic projection coordinates is derived in Ref. 48,

$$
\begin{aligned}
V_{i j}^{(D D)}= & \frac{\mu_{D}^{2}}{4 \pi \epsilon_{0} r_{i j}^{3}}\left\{\frac{16 R^{2}\left(\delta_{\mu \nu} \xi_{i}^{\mu} \xi_{j}^{\nu}\right)+\left(\sigma_{i}-4 R^{2}\right)\left(\sigma_{j}-4 R^{2}\right)}{\left(\sigma_{i}+4 R^{2}\right)\left(\sigma_{j}+4 R^{2}\right)}\right. \\
& +3 \frac{4 R\left(x_{i}-x_{j}\right) \xi_{i}^{1}+4 R\left(y_{i}-y_{j}\right) \xi_{i}^{2}+\left(z_{i}-z_{j}\right)\left(\sigma_{i}-4 R^{2}\right)}{\left(\sigma_{i}+4 R^{2}\right)} \\
& \left.\times \frac{4 R\left(x_{i}-x_{j}\right) \xi_{j}^{1}+4 R\left(y_{i}-y_{j}\right) \xi_{j}^{2}+\left(z_{i}-z_{j}\right)\left(\sigma_{j}-4 R^{2}\right)}{\left(\sigma_{j}+4 R^{2}\right)}\right\} .
\end{aligned}
$$

The last term in Eq. (42) is the constraining potential used to evaluate the integrals in Eq. (27),

$$
V_{c}=\sum_{i=1}^{n}\left(\frac{\left|\mathbf{r}_{i}-\mathbf{R}_{\mathrm{CM}}\right|}{2 R_{C}}\right)^{20},
$$

where $\mathbf{r}_{i}$ is the location of particle $i, \mathbf{R}_{\mathrm{CM}}$ is the location of the center of mass of the cluster, and $R_{C}$ is a parameter. The configuration space is curved and for $n$ Stokmayer point particles is made up of the direct product of $n \mathrm{R}^{3}$ spaces for the motion of the centers, and $n \mathrm{~S}^{2}$ spaces, for the orientation of the ideal dipole moments.

$$
\mathrm{M}=\frac{\mathbb{R}^{3} \otimes \mathbb{R}^{3} \otimes \cdots \mathrm{R}^{3}}{n \text { times }} \otimes \frac{\mathrm{S}^{2} \otimes \mathrm{S}^{2} \otimes \cdots \mathrm{S}^{2}}{n \text { times }}
$$

$\mathrm{M}$ is the same space that would be required to treat $n$ rigid rotors. The metric tensor in $\mathbb{M}$ is diagonal,

$$
g_{\mu \nu}=\operatorname{diag}\{\underbrace{m, \ldots, \mu_{\delta}}_{3 n \text { times }}\left(\frac{4 R^{2}}{\sigma_{1}+4 R^{2}}\right)^{2}, \ldots, \mu_{\delta}\left(\frac{4 R^{2}}{\sigma_{n}+4 R^{2}}\right)^{2}, \underbrace{\mu_{\delta}\left(\frac{4 R^{2}}{\sigma_{1}+4 R^{2}}\right)^{2}, \ldots, \mu_{\delta}\left(\frac{4 R^{2}}{\sigma_{n}+4 R^{2}}\right)^{2}}_{n \text { times }}\} .
$$

$m$ is the mass associated with the centers (total mass) and $\mu_{\delta}$ is the reduced mass associated with the rotations of the centers. The square root of the determinant of the metric tensor is

$$
g^{1 / 2}=\left(m^{3 n} \mu_{\delta}^{2 n}\right)^{1 / 2} \prod_{i=1}^{n}\left(\frac{4 R^{2}}{\sigma_{i}+4 R^{2}}\right)^{2} .
$$
form,

The Laplace-Beltrami operator takes a relatively simple

$$
\Delta_{\mathrm{LB}}=g^{\mu \nu} \partial_{\mu} \partial_{\nu}
$$

where $g^{\mu \nu}$ is the inverse of the metric tensor, which can be obtained trivially from Eq. (47), and the double sum in Eq. (49) is over the $5 n$ dimensions. The values of the parameters used in this study are in Table I, together with the binding energy of the dimer, and the energy of the two minima for the trimer, $E_{0}$ and $E_{1}$, a ring and a chain, respectively. The parameters are chosen to reproduce $\mathrm{HCl}$ aggregates, within the accuracy of the Stokmayer model. The minimization of the trimer is carried out with the Brownian algorithm developed for curved spaces. ${ }^{48}$ Figure 6 contains the energies estimated for the trimer on two separate $x$-axis scales. The upper scale is associated with the curves represented with black and white squares. These data sets are variational energies obtained by evaluating the integrals in Eq. (27) with Metropolis (white squares) and parallel tempering (black squares). ${ }^{37-40}$ The energies are obtained for a number of different values of the parameter $\beta$. The top $x$ axis of Fig. 6 is $T=\left(k_{B} \beta\right)^{-1}$ in $\mathrm{K}$. The simulations by Metropolis and parallel tempering are carried out by performing a $1 \times 10^{6}$ move walk to reach the asymptotic distribution, followed by a million moves to average the energy. The entire processes is repeated ten independent times, and the mean values for each sample are averaged and processed further to obtain a $95 \%$ confidence interval represented by the error bars. The two curves are significantly different at all values of $T$, indicating that quasiergodicity plays a role. The optimal value of $T$ is 44.6 K obtained from the parallel tempering data. The DMC simulations consist of GFIS-SPDMC runs at several values of $\Delta \tau$. The convergence of the GFIS-SPDMC estimate of the energy with respect to $\Delta \tau$ is evidenced by the dark circles in 
TABLE I. Parameters and results for the $\mathrm{LJDD}_{3}$ cluster.

\begin{tabular}{lcc}
\hline \hline Parameter & Symbol & Value (a.u.) \\
\hline Dipole moment & $\mu_{D}$ & 0.46 \\
L-J energy scale & $\epsilon$ & 0.001899 \\
L-J length scale & $r_{0}$ & 6.546 \\
Dipole size & $R$ & 2.44 \\
Mass of the center & $m$ & 65600 \\
Reduced mass & $\mu_{\delta}$ & 1790 \\
Constraining radius & $R_{C}$ & 5.32 \\
Binding energy of the dimer & $D_{0}$ & 0.00300 \\
$D_{3 h}$ minimum energy & $E_{0}$ & -0.007739 \\
$D_{\infty h}$ minimum energy & $E_{1}$ & -0.006210 \\
Opt. variational parameter & $\beta$ & 7077 \\
Opt. variational energy & $\left\langle E_{\beta}\right\rangle$ & $-0.006268 \pm 0.000002$ \\
DMC energy & $\langle E\rangle$ & $-0.006315 \pm 0.000005$ \\
\hline \hline
\end{tabular}

Fig. 6, where the bottom $x$-axis is $\Delta \tau$. The GFIS-SPDMC data are obtained by averaging ten independent runs; each run contains 200 iterations of a population with $N \approx 10^{5}$ replicas. We verify that the first 100 iterations are more than sufficient to achieve the asymptotic convergence, and the remaining iterations are used to average the energy. The error bars represent an estimate of the $95 \%$ confidence interval, obtained with the block averaging procedure detailed earlier. The ground state energies estimated variationally and by diffusion are both below $E_{1}$, by a statistically significant amount, making the $D_{\infty h}$ configuration of the trimer classically forbidden.

One useful characteristic of a configuration is the magnitude of the net dipole moment. This quantity depends on the vector sum of all $n$ dipole moments associated with the centers. We compare the size of the dipole vector with the maximum size it can attain, namely, $n \mu_{D}$. The ratio of these two quantities is symbolized as $|\mathbf{D}|$, and this can be readily computed from the stereographic projection coordinates of all the centers,

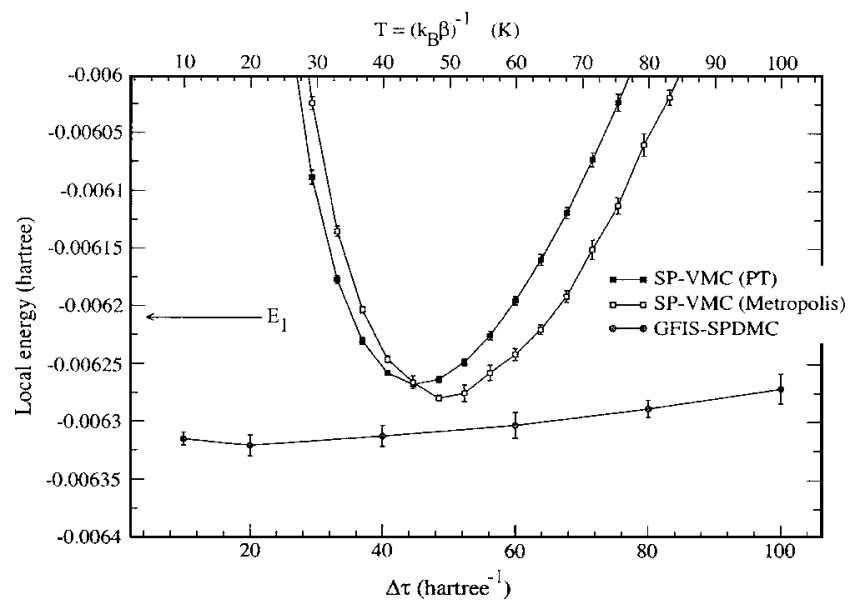

FIG. 6. Composite graph of VMC (top $x$ axis) and DMC (bottom $x$ axis) estimates for the ground state energy of the LJDD trimer. The white and black squares are the variational energies with $\psi_{T}=\exp \left(-V / 2 k_{B} T\right)$ as a trial function for several values of $T$ obtained using Eq. (27) with Metropolis and parallel tempering, respectively. The dark circles are the GFIS-SPDMC results for several values of $\Delta \tau$. The line marked $\mathrm{E}_{1}$ refers to the energy of the straight chain. [cf. Table I for a more precise value].

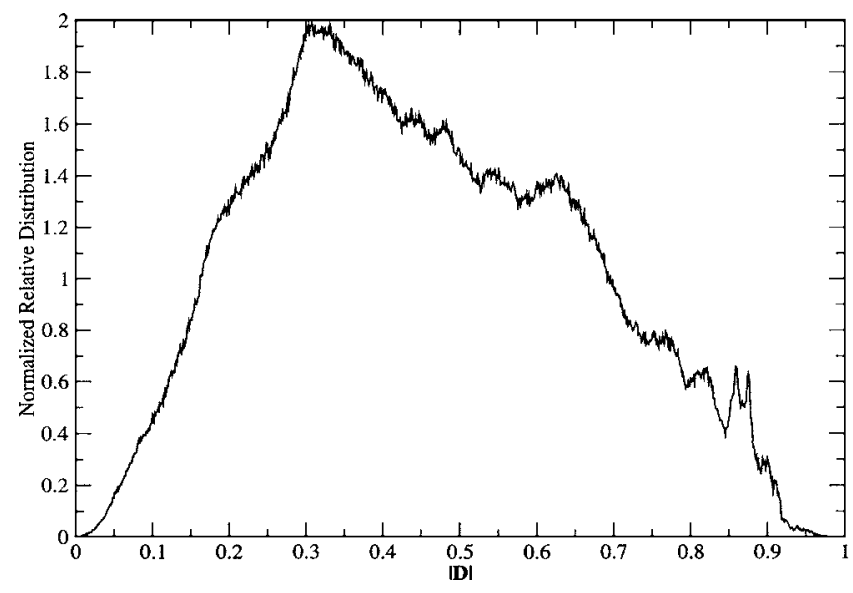

FIG. 7. A graph of the distribution of the relative dipole moment obtained with GFIS-SPDMC for the LJDD trimer [cf. Eq. (50)].

$$
\begin{aligned}
|\mathbf{D}|= & \frac{1}{n}\left[\left(\sum_{i=1}^{n} \frac{4 R \xi_{i}^{1}}{\sigma_{i}+4 R^{2}}\right)^{2}+\left(\sum_{i=1}^{n} \frac{4 R \xi_{i}^{2}}{\sigma_{i}+4 R^{2}}\right)^{2}\right. \\
& \left.+\left(\sum_{i=1}^{n} \frac{\sigma_{i}-4 R^{2}}{\sigma_{i}+4 R^{2}}\right)^{2}\right]^{1 / 2} .
\end{aligned}
$$

The structural parameter $|\mathbf{D}|$ can have a value between 0 , for a symmetric structure like a ring, to 1 for a straight chain. The quantity $|\mathbf{D}|$ is, in essence, a type of rotationally invariant bond orientational parameter. We have made extensive use of $|\mathbf{D}|$ to characterize random walks of anisotropic systems in recent works. ${ }^{3}$ In Fig. 7 we plot the distribution of |D $\mid$ obtained by collecting its values for every replica, for 100 iterations following the usual 100 iterations to assure asymptotic behavior. The simulation produces 10015578 estimates for $|\mathbf{D}|$ which are tallied into 1000 bins between 0 and 1. The mean, standard deviation, and skewness are $0.4519,0.20119$ and 0.2495 , respectively.

\section{CONCLUSIONS}

In this article we develop and test several importance sampling schemes for diffusion Monte Carlo in curved spaces mapped projectively. We find the equivalent of the Fokker-Plank diffusion equation and the equivalent of Green's function propagator with IS. Aside from the difference in generating the diffusion steps, ${ }^{1}$ the latter scheme is identical to its equivalent in flat space mapped by Cartesian coordinates; as such, it is the most convenient to implement in non-Euclidean spaces, in general. Both importance sampling schemes rely on the availability of optimized trial wavefunctions. We propose and test a family of convenient trial wavefunctions and a method based on parallel tempering to optimize them in curved spaces while eliminating possible numerical difficulties with the DMC sampling. Applications of these methods to clusters of rigid molecules are likely to run into complex and glassylike potential energy surfaces with numerous barriers. While DMC, especially with IS is known to allow tunneling, we use the double well quartic potential in one-dimensional flat space to systematically explore the behavior of guided and unguided DMC simulations. We find the unguided DMC simulations begin- 
ning from the incorrect well can either take a long time or become trapped altogether even in classically allowed minima. We present evidence that confirms the conventional understanding of how IS-DMC can overcome sampling problems. Namely, initiating the walk from an optimized variational distribution and performing few diffusionbranching steps reshapes the trial wavefunction efficiently. Using parallel tempering and the thermal distribution we propose in this work is a way to improve one's confidence that all the important configurations of the potential energy surface are included in the starting distribution. Alternatively, one could exploit the information provided in Fig. 1(a) to build a better initial distribution of replica. In fact, it is evident that using both long time steps and low masses the system appears able to surmount the potential barrier separating the two wells. Thus, after an initial simulation stage where unphysical masses or inertia moments are used in conjunction with large $\Delta \tau$, one could slowly reduce the former to make the replica distribution approaching the exact one. Clearly, the possibility of using longer time steps thanks to improved DMC schemes facilitates the sampling of topologically complicated potential energy surfaces ${ }^{50}$ even further. A similar idea was previously exploited in the context of global optimization by Finnila et al. ${ }^{51}$ who showed the suitability of this approach to locate lowest energy isomers for LennardJones clusters containing up to 19 atoms.

With the particle in a ring we confirm that both the adapted Fokker-Plank diffusion equation and the equivalent of Green's function propagator with importance sampling accomplish the following in curved spaces mapped projectively: (a) improve the efficiency of unguided DMC simulations; (b) improve the convergence behavior of unguided DMC simulations, and with it its accuracy; (c) overcome sampling problems that arise from the nonconfining nature of the potential energy. These features are crucial for future applications that we have in mind for GFIS-SPDMC in molecular clusters. We demonstrate the applicability of GFISSPDMC by computing the ground state energy and wavefunction of the LJDD trimer. It is evident in Fig. 6 that parallel tempering moves affect the thermal distributions and consequently the variational estimates of the ground state energy over the range of values of $T$. The optimized "thermal" wavefunction oversamples the classically forbidden chain configuration. However, we can expect that in approximately $50-100$ hartree $^{-1}$, as Fig. 4 suggests, the branching part of GFIS-SPDMC adjusts the wavefunction to its exact shape. This total diffusion time corresponds to about ten diffusion steps for the smallest value of $\Delta \tau$ used for the simulation of the LJDD trimer.

\section{ACKNOWLEDGMENTS}

Acknowledgment is made to the donors of the Petroleum Research Fund, administered by the ACS (Grant No. 40946B6) for partial support of this research. This work has also been supported, in part, by the National Science Foundation (Grant No. CHE0554922). Support is also gratefully acknowledged from The Stacy Ann Vitetta ' 82 Professorship Fund, and The Ellington Beavers Fund for Intellectual Inquiry from Arcadia University. M.M. acknowledges an EPSRC Advanced Research Fellowship (GR/R77803/01).

${ }^{1}$ M. W. Avilés and E. Curotto, J. Phys. Chem. A 111, 2610 (2007).

${ }^{2}$ S. F. Langley, E. Curotto, D. L. Freeman, and J. D. Doll, J. Chem. Phys. 126, 084506 (2007).

${ }^{3}$ M. W. Avils, P. T. Gray, and E. Curotto, J. Chem. Phys. 124, 174305 (2006).

${ }^{4}$ M. F. Russo, Jr. and E. Curotto, J. Chem. Phys. 120, 2110 (2004).

${ }^{5}$ M. F. Russo, Jr. and E. Curotto, J. Chem. Phys. 118, 6806 (2003).

${ }^{6}$ J. B. Anderson, J. Chem. Phys. 63, 1499 (1975).

${ }^{7}$ P. Håkansson, M. Mella, D. Bressanini, G. Morosi, and M. Patrone, J. Chem. Phys. 125, 184106 (2006).

${ }^{8}$ P. Håkansson and M. Mella, J. Chem. Phys. 126, 104106 (2007).

${ }^{9}$ M. Mella and D. C. Clary, J. Chem. Phys. 119, 10048 (2003).

${ }^{10}$ M. Mella, G. Morosi, and D. Bressanini, Phys. Rev. E 61, 2050 (2000).

${ }^{11}$ P. J. Reynolds, D. M. Ceperley, B. J. Alder, and W. A. Lester, Jr., J. Chem. Phys. 77, 5593 (1982).

${ }^{12}$ D. M. Ceperley and B. J. Alder, J. Chem. Phys. 81, 5834 (1984).

${ }^{13}$ D. M. Ceperley and B. J. Alder, Science 231, 555 (1986).

${ }^{14}$ M. H. Kalos, D. Levesque, and L. Verlet, Phys. Rev. A 9, 2178 (1974).

${ }^{15}$ D. M. Arnow, M. H. Kalos, M. A. Lee, and K. E. Schmidt, J. Chem. Phys. 77, 5562 (1982).

${ }^{16}$ C. J. Umrigar, M. P. Nightingale, and K. J. Runge, J. Chem. Phys. 99, 2865 (1993)

${ }^{17}$ A. Sarsa, K. E. Schmidt, and J. W. Moskowitz, J. Chem. Phys. 113, 44 (2000).

${ }^{18}$ D. C. Clary, J. Chem. Phys. 114, 9725 (2001).

${ }^{19}$ H. Jiang, M. Z. Xu, J. M. Hutson, and Z. Baçìc, J. Chem. Phys. 123, 054305 (2005).

${ }^{20}$ H. Jiang and Z. Baçìc, J. Chem. Phys. 122, 244306 (2005).

${ }^{21}$ F. Paesani, F. A. Gianturco, and K. B. Whaley, J. Chem. Phys. 115, 10225 (2001).

${ }^{22}$ J. W. Moskowitz, Z. Baçìc, A. Sarsa, and K. E. Schmidt, J. Chem. Phys. 114, 10294 (2001)

${ }^{23}$ V. Melik-Alaverdian, N. E. Bonesteel, and G. Ortiz, Phys. Rev. Lett. 79, 5286 (1997).

${ }^{24}$ B. A. Schutz, First Course in General Relativity (Cambridge, New York, 1985).

${ }^{25}$ W. D. Curtis and F. R. Miller, Differential Manifolds and Theoretical Physics (Academic, New York, 1985).

${ }^{26}$ H. Kleinert, Path Integrals in Quantum Mechanics, Statistics and Polymer Physics (World Scientific, Singapore, 1990).

${ }^{27}$ B. S. DeWitt, Rev. Mod. Phys. 29, 377 (1957).

${ }^{28}$ M. M. Mizrahi, J. Math. Phys. 16, 2201 (1975).

${ }^{29}$ W. A. Lester, Jr., and B. L. Hammond, Annu. Rev. Phys. Chem. 41, 283 (1990).

${ }^{30}$ V. Buch, J. Chem. Phys. 97, 726 (1992).

${ }^{31}$ M. Lewerenz, J. Chem. Phys. 104, 1028 (1996).

${ }^{32}$ H. Sun and R. O. Watts, J. Chem. Phys. 92, 603 (1990).

${ }^{33}$ P. Sandler, J. Oh Jung, M. M. Szxzçśniak, and V. Buch, J. Chem. Phys. 101, 1378 (1994)

${ }^{34}$ H.-S. Lee, J. M. Herbert, and A. B. McCoy, J. Chem. Phys. 110, 5481 (1999).

${ }^{35}$ P. Sandler, V. Buch, and J. Sadlej, J. Chem. Phys. 105, 10387 (1996).

${ }^{36}$ M. W. Avilés and E. Curotto, J. Chem. Phys. 122, 164109 (2005).

${ }^{37}$ M. Falcioni and M. W. Deem, J. Chem. Phys. 110, 1754 (1999).

${ }^{38}$ J. P. Neirotti, F. Calvo, D. L. Freeman, and J. D. Doll, J. Chem. Phys. 112, 10340 (2000)

${ }^{39}$ F. Calvo, J. P. Neirotti, D. L. Freeman, and J. D. Doll, J. Chem. Phys. 112, 10350 (2000).

${ }^{40}$ J. P. Neirotti, D. L. Freeman, and J. D. Doll, Phys. Rev. E 62, 7445 (2000).

${ }^{41}$ D. D. Frantz, D. L. Freeman, and J. D. Doll, J. Chem. Phys. 93, 2769 (1990).

${ }^{42}$ D. T. Colbert and W. H. Miller, J. Chem. Phys. 96, 1982 (1992).

${ }^{43}$ J. Bartke and R. Hentschke, Phys. Rev. E 75, 061503 (2007).

${ }^{44}$ B. Groh and S. Dietrich, Phys. Rev. E 63, 021203 (2001).

${ }^{45}$ I. P. Omelyan, I. M. Mryglod, and M. V. Tokarchuk, Phys. Rev. E 51, 5976 (1995).

${ }^{46}$ M. J. Stevens and G. S. Grest, Phys. Rev. E 51, 5976 (1995). 
${ }^{47}$ K.-K. Han, J. H. Cushman, and D. J. Diestler, J. Chem. Phys. 96, 7867 (1992).

${ }^{48}$ C. A. Oppenheimer and E. Curotto, J. Chem. Phys. 121, 6226 (2004).

${ }^{49}$ D. M. Pav and E. Curotto, J. Chem. Phys. 123, 144301 (2005).
${ }^{50}$ S. Chiesa, M. Mella, G. Morosi, and D. Bressanini, J. Chem. Phys. 99, 8225 (2003).

${ }^{51}$ A. B. Finnila, M. A. Gomez, C. Sebenik, C. Stenson, and J. D. Doll, Chem. Phys. Lett. 219, 343 (1994). 